\title{
Aircraft Fault Detection Using Real-Time Frequency Response Estimation
}

\author{
Jared A. Grauer* \\ NASA Langley Research Center, Hampton, Virginia 23681
}

\begin{abstract}
A real-time method for estimating time-varying aircraft frequency responses from input and output measurements was demonstrated. The Bat-4 subscale airplane was used with NASA Langley Research Center's AirSTAR unmanned aerial flight test facility to conduct flight tests and collect data for dynamic modeling. Orthogonal phase-optimized multisine inputs, summed with pilot stick and pedal inputs, were used to excite the responses. The aircraft was tested in its normal configuration and with emulated failures, which included a stuck left ruddervator and an increased command path latency. No prior knowledge of a dynamic model was used or available for the estimation. The longitudinal short period dynamics were investigated in this work. Time-varying frequency responses and stability margins were tracked well using a 20 s sliding window of data, as compared to a post-flight analysis using output error parameter estimation and a low-order equivalent system model. This method could be used in a real-time fault detection system, or for other applications of dynamic modeling such as real-time verification of stability margins during envelope expansion tests.
\end{abstract}

\section{Nomenclature}

sinusoid amplitude

vertical acceleration, $g$ units

wingspan, $\mathrm{ft}$

wing mean aerodynamic chord, ft

frequency response

acceleration due to gravity, $\mathrm{ft} / \mathrm{s}^{2}$

altitude, $\mathrm{ft}$

inertia tensor elements, slug. $\mathrm{ft}^{2}$

imaginary number, $\sqrt{-1}$

gain

aerodynamic lift force, lbf

aerodynamic pitch moment, ft.lbf

mass, slug

number of samples

pitch rate, $\mathrm{rad} / \mathrm{s}$

wing reference area, $\mathrm{ft}^{2}$

Laplace variable

record length, s

time constant, $1 / \mathrm{s}$

time, $\mathrm{s}$

$\begin{array}{ll}t_{w} & \text { window length, } \mathrm{s} \\ u & \text { input variable } \\ V & \text { true airspeed, } \mathrm{ft} / \mathrm{s} \\ y & \text { output variable } \\ \alpha & \text { angle of attack, rad } \\ \Delta & \text { change or perturbation } \\ \delta & \text { control input } \\ \zeta & \text { damping ratio } \\ \tau & \text { time delay, } \mathrm{s} \\ \phi & \text { phase angle, } \mathrm{rad} \\ \omega & \text { frequency, rad } / \mathrm{s}\end{array}$

Subscripts

$\begin{array}{ll}\mathrm{cm} & \text { center of mass } \\ \text { lat } & \text { lateral } \\ \text { lon } & \text { longitudinal } \\ \text { ped } & \text { pedal } \\ \text { sp } & \text { short period } \\ x, y, z & \text { body axes }\end{array}$

*Research Engineer, Dynamic Systems and Control Branch, MS 308. Senior Member AIAA. 


\section{Introduction}

$\mathrm{H}^{\text {Aults }}$ in aircraft subsystems can result in catastrophic failures. A review of 126 aircraft accidents in $\mathrm{F}$ which loss-of-control was a factor ${ }^{1}$ found that $45 \%$ of the cases incurred system faults, failures, and errors, and that $18 \%$ of the cases included vehicle damage. Identifying and addressing these factors is therefore important for improving flight safety. Modern flight control systems typically employ redundant subsystems to help reduce single-point failures, and fault-detection algorithms to alert the crew.

One problem that can arise when attempting to detect faults is the need for an accurate dynamic model of the aircraft. This may be difficult because faults can be complex to model, exist in varying degrees, and occur in multiple combinations. One solution, for example Refs. [2,3], is to use a Kalman filter for each fault considered, resulting in a large bank of filters run in parallel during flight.

A different approach is to employ non-parametric modeling techniques. This can be advantageous because only input and output variable measurements are needed, and no dynamic model is assumed. One technique is to use direct adaptive neural-networks to identify the input-output dynamic relationship, as was done in Ref. [4] with a model-following controller that improved handling qualities during a stabilator fault. Another non-parametric technique is to use frequency responses, which are often computed in an off-line environment using spectral density estimates, where averaging and windowing can be leveraged to reduce errors. One work-around towards real-time estimation has been to process data using onboard computers or a ground station immediately after a flight test maneuver in a quasi real-time fashion, while the aircraft is still in flight. ${ }^{5,6}$ Other studies used simulations ${ }^{7}$ and piloted simulations ${ }^{8,9}$ to demonstrate time-varying frequency response estimation with discrete wavelet transforms. Another study ${ }^{10}$ analyzed batch windows of data sequentially with the discrete Fourier transform to estimate frequency responses.

In a related work, Grauer and Morelli ${ }^{11}$ presented a simple non-parametric method for computing frequency responses in real time. Orthogonal phase-optimized multisine inputs were used to excite all axes of the aircraft simultaneously, and a recursive Fourier transform was employed to continuously update frequencydomain data, which were used to compute frequency responses and stability margins. A sliding window of data facilitated the identification of time-varying dynamics, such as during aircraft faults.

This paper presents a flight test of an airplane using the method in Ref. [11] for dynamic modeling during in-flight simulated faults. Three cases are considered here: the first is straight and level flight without any failures, the second includes a stuck ruddervator control surface, and the third includes an added command path latency. The aircraft and flight test are described in Section II. Methods for the data analysis are given in Section III. Estimation results are presented in Section IV, and are further elaborated upon in Section V. Section VI draws concluding remarks.

Software for the input design, post-flight data compatibility analysis, and the equation error and output error parameter estimation used in this work are available in a MATLAB ${ }^{\circledR}$ toolbox called System IDentification Programs for AirCraft, or SIDPAC. ${ }^{12}$

\section{Flight Test Experiment}

\section{II.A. Bat-4 Airplane}

The aircraft flown was the subscale Bat-4 airplane, tail number N105BT. The Bat-4 is a fixed-wing, remotely-piloted, unmanned aircraft. It has fixed tricyle landing gear, a twin-boom tail section, a single fixed-pitch pusher propeller, and a $6.6 \mathrm{in}^{3}$ two-stroke gasoline engine. It is manufactured by the MLB Company and marketed as an aerial mapping, surveillance, and payload platform. Its maximum speed is $70 \mathrm{kt}$, and cruise speed is about $50 \mathrm{kt}$. Figure 1 shows a photograph of the Bat- 4 during the flight test, as well as a three-view technical drawing. Geometry and nominal mass properties of the airplane are given in Table 1.

The Bat-4 has controls for throttle, flaps, ailerons, and ruddervators. Each control surface can be actuated independently, for research purposes. Pilot inputs were passed through command-shaping filters and were added to trim values set by the pilot to generate conventional elevator, aileron, and rudder commands. The aileron command was also fed through to the pedal command shaper to help coordinate turns. These commands were then allocated to the different control surfaces, and passed through position and rate limiters before being sent to the actuators. Pilot inputs moved ailerons asymmetrically to control roll moments, and ruddervators both symmetrically and asymmetrically to control pitch and yaw moments, respectively. The sign convention is that positive pilot inputs (stick forward, stick left, left pedal down) create negative angular 


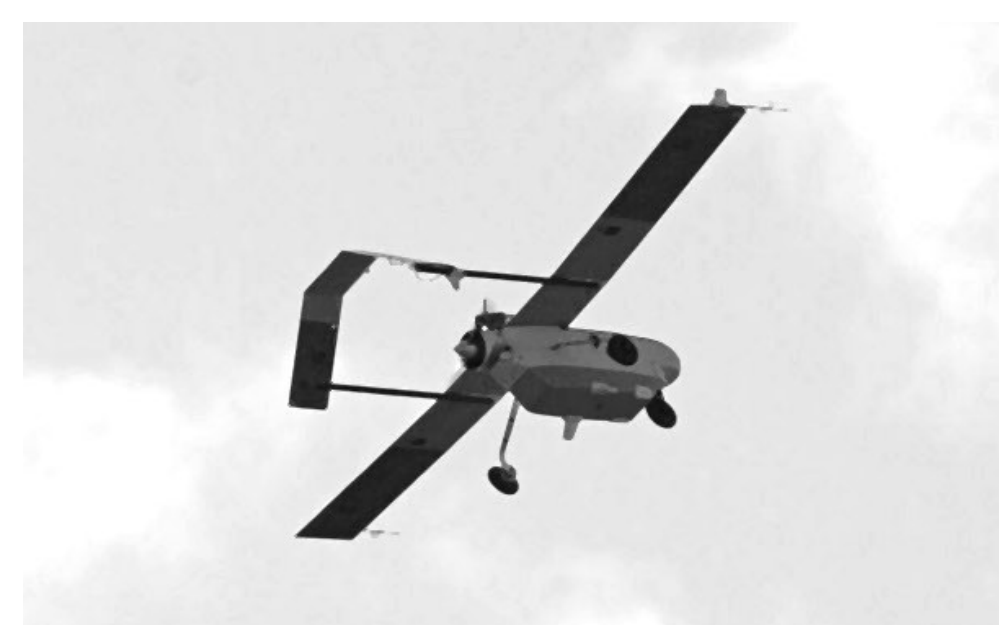

(a) flight over Wallops Island (credit: NASA Wallops Flight Facility)

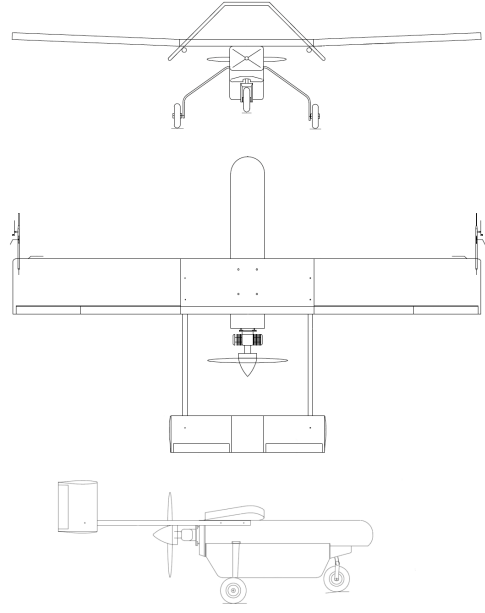

(b) three-view drawing

Figure 1. Bat-4 airplane

rates (nose down, right wing up, nose left). The airplane was flown open loop, without any feedback control.

The airplane was fit with custom hardware and sensors for research flight testing. Onboard sensors included but were not limited to rate gyros, linear accelerometers, outside air temperature sensors, GPS, and estimated Euler angles. Vanes on dual wing-tip mounted air-data booms provided angle of attack and sideslip angle measurements. Pressure taps on the booms also delivered static and total pressures, from which airspeed and altitude were computed. Fuel flow measurements were combined with a CAD model of the aircraft and a pre-flight weight and balance test to model the changing mass properties during flight. All measurements were sampled and transmitted to the ground at $200 \mathrm{~Hz}$.

\section{II.B. Operation}

The data in this work is from a single flight of the Bat-4, conducted on April 16, 2015 at NASA Wallops Flight Facility, on Wallops Island, Virginia. The vehicle was flown using the NASA Langley AirSTAR (Airborne Subscale Transport Aircraft Research) flight test facility. A research pilot flew the aircraft from inside a ground control station using a synthetic vision display drawn from telemetry data and a local terrain database, along with video from a camera mounted in the nose of the aircraft. Pilot inputs and other commands were transmitted to the aircraft flight control system via a command and control telemetry link. References $[13,14]$ provide additional details about the flight test and the AirSTAR flight test facility.

Research maneuvers were executed by the pilot flying to a specified flight condition and applying computer-generated inputs, referred to as wavetrains. These inputs were added either to control surface deflection commands just upstream of the servos, or to the pilot stick and pedal commands just upstream of the command shapers. To accommodate other test objectives, wavetrains in this flight were stored onboard the aircraft instead of uplinked from the control station. Wavetrains were engaged by the pilot pushing and holding a button on the thrust lever. Similarly, simulated faults were engaged by the flight test engineer engaging switches inside the control station. This setup has proven invaluable for flight testing feedback control and system identification technologies in a rapid and cost-efficient manner.

Inside the control station, flight data downsampled to $50 \mathrm{~Hz}$ was available to research engineers through the internal ethernet connections. An existing graphical user interface, ${ }^{15}$ written in MATLAB ${ }^{\circledR}$, was modified for computing and viewing real-time frequency response estimates. An example screenshot is shown in Fig. 2. The first column shows input and output perturbation signals, the second column shows Fourier transforms of the data, and the third column shows estimated frequency response and stability margin estimates. 


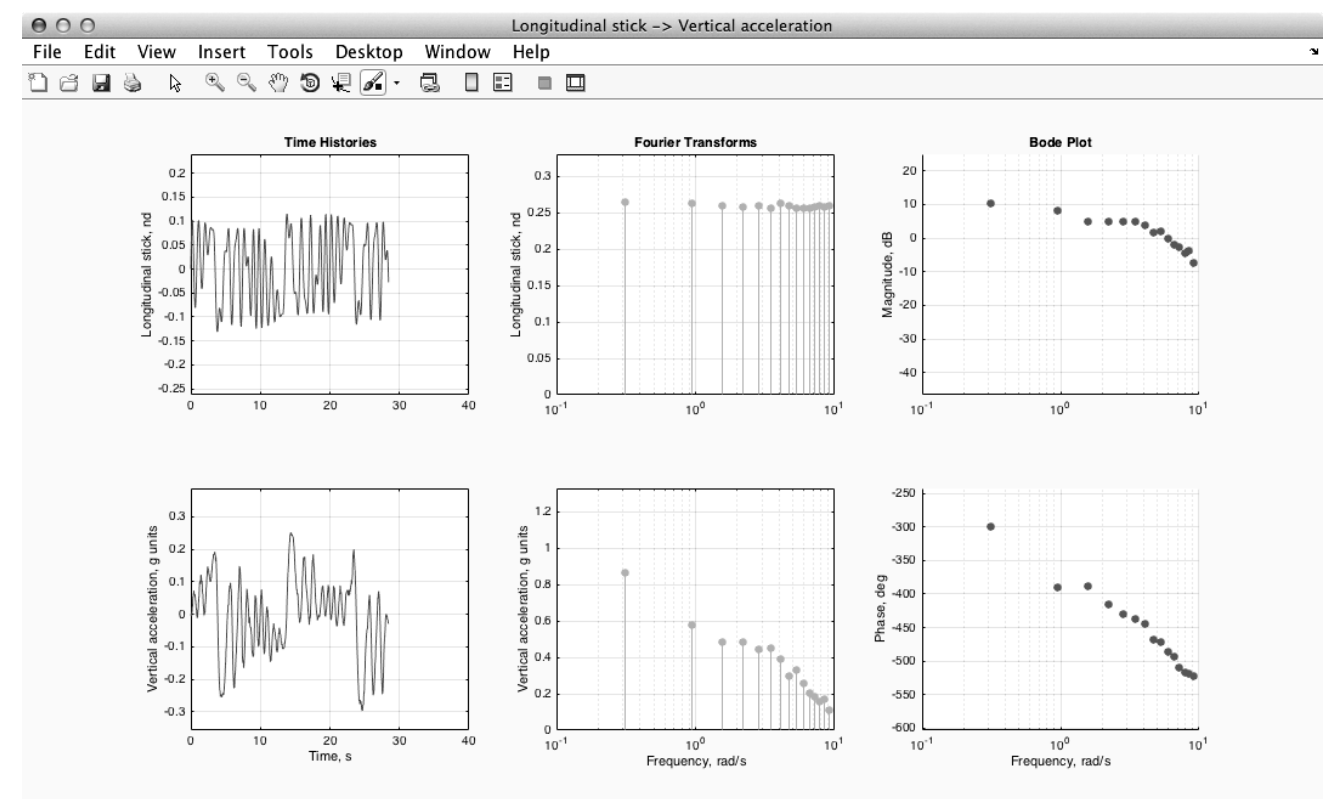

Figure 2. Screenshot of a real-time display

\section{II.C. Maneuvers}

Three of the research maneuvers completed in this flight are examined in this work. Each of these three maneuvers began in straight and level trimmed flight, and included a $40 \mathrm{~s}$ wavetrain input added to the pilot stick inputs. The wavetrain was an orthogonal phase-optimized multisine. These inputs were developed at NASA Langley ${ }^{16,17}$ and have been used successfully in flight conditions such as high sideslip, stall and post-stall, hypersonic, and normal flight; in hazardous weather such as in-flight icing and turbulence; and for vehicles including airplanes, rotorcraft, and aeroelastic vehicles. Each multisine input has the form

$$
u(t)=a \sum_{k} a_{k} \sin \left(\frac{2 \pi k}{T} t+\phi_{k}\right)
$$

where $a$ is the aggregate amplitude, $a_{k}$ are the sinusoid amplitudes, $T$ is the excitation record length, and $\phi_{k}$ are the phase angles. Choice of the record length determines the excitation frequencies $\omega_{k}=2 \pi k / T$. Selection of the sinusoid amplitudes can be used to tailor the power spectrum of the inputs. Phase angles are optimized for minimium relative peak factor (RPF) of the sum $u(t)$, which creates small perturbation responses. The aggregate amplitude is used to scale the resulting waveform to achieve good signal-to-noise ratios.

During past experiments with AirSTAR, requirements on range restricted wavetrains to $10 \mathrm{~s}$ in duration. Because the test volume was expanded in this experiment, the wavetrain record length was increased to $40 \mathrm{~s}$ to include more time on condition and significantly lower excitation frequencies. This duration corresponded to a resolution of $0.025 \mathrm{~Hz}$ for the excitation frequencies, which was finer than in previous experiments. The input bandwidth was selected as $0.050 \mathrm{~Hz}$ to $1.525 \mathrm{~Hz}$ to encompassed the expected rigid body modes of interest. Excitation frequencies were assigned in an alternating manner amongst four inputs, so that each input had a wide-band spectrum and 15 excitation frequencies. In this work only three of those four designed inputs were used, and these were summed with the pilot longitudinal stick, lateral stick, and pedal inputs. The longitudinal stick input was assigned the multisine with the lowest excitation frequency to help excite the phugoid mode, which was not possible in previous experiments with AirSTAR. Because prior information on the aircraft dynamics was not available, a uniform power spectrum which excited each frequency equally was chosen. Flight test results showed that the phugoid mode was indeed well excited, and ramifications of this for frequency response estimation are discussed later. Ten wavetrains, with different combinations of "low" and "high" aggregate amplitudes, were stored in the aircraft onboard flight computer. During a brief in-flight trial-and-error procedure, the combination referred to as Wavetrain 21 was selected for the remaining maneuvers. This wavetrain is shown in Fig. 3 and parameterized in Table 2. Pilot stick and 
pedal deflections measurements are normalized by their maximum values, so that each of these inputs have amplitude equal to approximately $10 \%$ of the available travel.

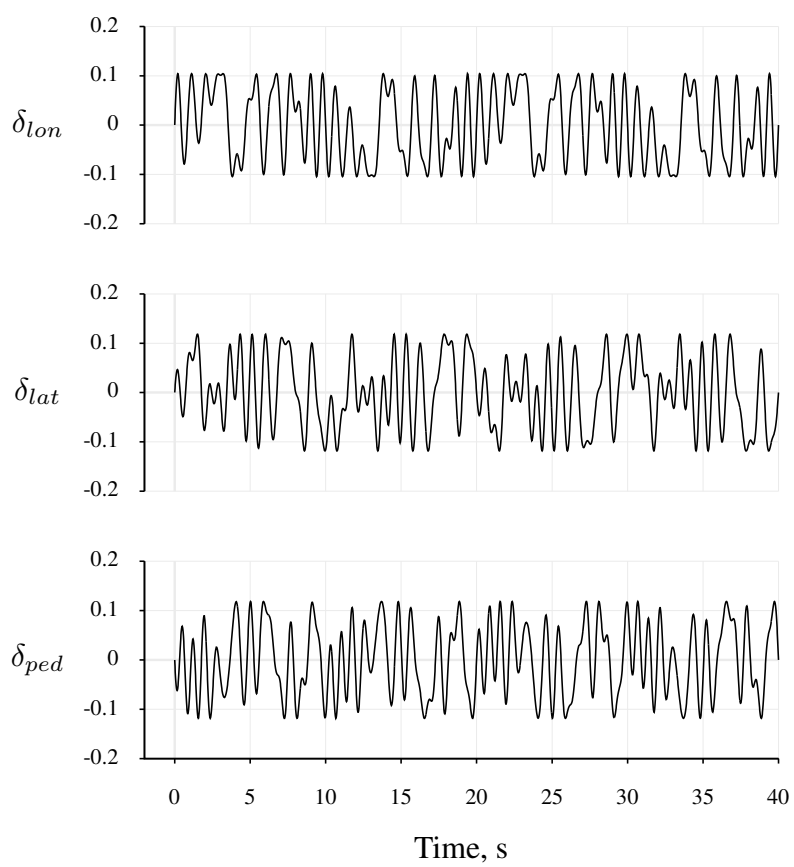

Figure 3. Multisine inputs, Wavetrain 21

\section{Methods}

\section{III.A. Model Forms}

Low-Order Equivalent System (LOES) models are employed to approximate nonlinear and higher-order aircraft responses from pilot inputs in terms of conventional aircraft modes and equivalent time delays. ${ }^{18,19,20}$ This is in contrast to bare-airframe models, where the inputs are the control surface deflections. These model structures are fixed and are usually restricted to the typical pilot input bandwidth $0.1 \mathrm{rad} / \mathrm{s}$ to $10 \mathrm{rad} / \mathrm{s}$. An example of a LOES model is the state-space equation for the short period approximation

$$
\begin{aligned}
{\left[\begin{array}{c}
\dot{\alpha}(t) \\
\dot{q}(t)
\end{array}\right] } & =\left[\begin{array}{cc}
-L_{\alpha} & 1 \\
M_{\alpha} & M_{q}
\end{array}\right]\left[\begin{array}{c}
\alpha(t) \\
q(t)
\end{array}\right]+\left[\begin{array}{c}
0 \\
M_{\delta_{l o n}}
\end{array}\right] \delta_{l o n}\left(t-\tau_{l o n}\right) \\
{\left[\begin{array}{c}
\alpha(t) \\
q(t) \\
a_{z}(t)
\end{array}\right] } & =\left[\begin{array}{cc}
1 & 0 \\
0 & 1 \\
-V L_{\alpha} / g & 0
\end{array}\right]\left[\begin{array}{l}
\alpha(t) \\
q(t)
\end{array}\right]+\left[\begin{array}{l}
0 \\
0 \\
0
\end{array}\right] \delta_{l o n}\left(t-\tau_{l o n}\right)
\end{aligned}
$$

In Eq. (2), states and controls represent perturbations from trim values, and the parameters that multiply them are assumed to be constants. The traditional terms $L_{q}$ and $L_{\delta_{l o n}}$ have been neglected, and effects due to actuators, filters, command shapers, etc. have been subsumed into the remaining terms. ${ }^{20}$

Equations (2) can be expressed as a transfer function matrix, by means of the Laplace transform and Cramer's rule, as

$$
\left[\begin{array}{c}
\frac{\alpha(s)}{\delta_{l o n}(s)} \\
\frac{q(s)}{\delta_{l o n}(s)} \\
\frac{a_{z}(s)}{\delta_{l o n}(s)}
\end{array}\right]=\frac{e^{-\tau_{l o n} s}}{s^{2}+\left(L_{\alpha}-M_{q}\right) s-\left(L_{\alpha} M_{q}+M_{\alpha}\right)}\left[\begin{array}{c}
M_{\delta_{l o n}} \\
M_{\delta_{l o n}} s+\left(L_{\alpha} M_{\delta_{l o n}}\right) \\
-V L_{\alpha} M_{\delta_{l o n}} / g
\end{array}\right]
$$


Because stability and control derivatives in LOES models describe different physics than in bare-airframe dynamics, it is often useful to write Eq. (3) using modal parameters, as

$$
\left[\begin{array}{c}
\frac{\alpha(s)}{\delta_{l o n}(s)} \\
\frac{q(s)}{\delta_{l o n}(s)} \\
\frac{a_{z}(s)}{\delta_{l o n}(s)}
\end{array}\right]=\frac{e^{-\tau_{l o n} s}}{s^{2}+2 \zeta_{s p} \omega_{s p}+\omega_{s p}^{2}}\left[\begin{array}{c}
k_{\dot{\theta}} \\
k_{\dot{\theta}}\left(s+1 / T_{\theta_{2}}\right) \\
k_{a_{z}}
\end{array}\right]
$$

\section{III.B. Recursive Fourier Transform}

Data in the time domain is converted into the frequency domain using the Fourier transform. Its definition, applied to the input signal over a finite period of time, is

$$
u(t)=\int_{0}^{T} u(t) e^{-j \omega t} d t
$$

For equally-spaced time samples of data and a set of discrete analysis frequencies, Eq. (5) can be approximated as

$$
u\left(j \omega_{k}\right)=\sum_{n=0}^{N-1} u\left(t_{n}\right) e^{-j \omega_{k} t_{n}} \Delta t
$$

Errors due to this approximation are negligible when the sampling rate is significantly higher than the frequencies of interest, as is the case here, although straightforward corrections can be applied.

Equation (6) is applied to data over the time interval $[0, T]$. Because it is a simple summation of terms, Eq. (6) can be manipulated to evidence its prior value at any time $t_{n}$ as

$$
u_{n}\left(j \omega_{k}\right)=u_{n-1}\left(j \omega_{k}\right)+u\left(t_{n}\right) e^{-j \omega_{k} t_{n}} \Delta t
$$

In this way, Fourier transforms are updated with information from each new measurement in a recursive fashion. These operations are simple and fast, consisting only of additions and multiplications, and can be performed for many signals and frequencies without much processing time.

\section{III.C. Real-Time Frequency Response Estimation}

Frequency responses can be computed from transfer function models, such as Eq. (3), by setting $s=j \omega$ and taking the form

$$
\frac{y(j \omega)}{u(j \omega)}=G(j \omega)
$$

Frequency responses can also be computed directly from measured steady-state response data, without requiring a transfer function model. Some methods for doing this include single-frequency sine-dwell tests, correlation methods, and spectral estimation methods. ${ }^{21,22,23}$

Another approach for frequency response estimation is to directly evaluate Eq. (8). This is done by transforming input and output data into the frequency domain using the Fourier transform, and then computing their ratios at frequencies where the input has sufficient power. This approach is sometimes referred to as the Empirical Transfer Function Estimate, ${ }^{22}$ though it was well established before this coinage. ${ }^{24}$ In general, this approach yields an inconsistent estimate of the frequency response because the steady-state variance of the estimates is equal to the corresponding signal-to-noise ratio at that frequency. Furthermore, estimates can be arbitrarily biased when the input, appearing in the denominator of Eq. (8), has low power. Consequently additional steps, such as windowing or binning, are typically applied to smooth these frequency response estimates. $^{22,25}$

However for periodic inputs, such as the multisine inputs described in Section II.C, Eq. (8) is an unbiased and consistent estimator of the frequency response, with errors proportional to $1 / N$. The input power exists only at a finite set of frequencies, which are the excitation frequencies and are known. Effects of measurement noise on the frequency response estimates are usually small because the response is only evaluated at the excitation frequencies, which have high signal-to-noise ratios. Because there are typically only modest 
numbers of excitation frequencies in most flight tests, computation requirements are small. Another benefit is that multisine inputs encourage the development of steady-state perturbation responses, which are sought in frequency response estimation. This is similar to the traditional single-input sine dwell tests, except that multiple frequencies on multiple different control effectors can be excited simultaneously. Another advantage of this approach is that no additional analyst judgment, for example choosing bin or window sizes used in other methods, is needed.

When the recursive Fourier transforms are used, for example Eq. (7), the frequency responses can be estimated in real time using this approach. Errors due to spectral leakage and partial-period evaluations of the Fourier transforms can be minimized by only updating the frequency response estimates at times that are integer multiples of the corresponding half period. Doing so increases the accuracy of the estimates and further decreases computational requirements. Because computations are not intensive, many frequency responses at many frequencies can be computed quickly and in real time. Stability margins can be computed from real-time frequency response estimates, which can help determine stability and detect changes in the input-output relationships.

\section{III.D. Adaptation and Data Forgetting}

As described so far, the real-time method uses the entire history of past data, up to the current sample, to compute the frequency response estimates. Changes in the physical process require enough new data to overwhelm past records and be reflected in the frequency response estimate. One approach to adapting to changing dynamics is to systematically forget past data in the analysis. In this way, the frequency response estimates can continuously adapt to current conditions in a straightforward manner.

There are several approaches to data forgetting; one method that has worked well with frequency response estimation is to use a sliding window of data. ${ }^{11}$ This is implemented with the input, for example, by appending an extra term to Eq. (7) as

$$
u_{n}\left(j \omega_{k}\right)=u_{n-1}\left(j \omega_{k}\right)+u\left(t_{n}\right) e^{-j \omega_{k} t_{n}} \Delta t-u\left(t_{n}-t_{w}\right) e^{-j \omega_{k}\left(t_{n}-t_{w}\right)} \Delta t
$$

Similar to the way that each new measurement adds a contribution to the Fourier transform, the last term in Eq. (9) then subtracts the contribution from a past measurement as it traverses the sliding window. This process uses only the last $t_{w}$ seconds of data in the real-time analysis. As there are no practical methods for selecting the data forgetting parameters, this is currently based on experience and simulation.

\section{Results}

In this section, the results of applying the real-time frequency response estimation method to three cases are presented. The first case was straight and level flight for the baseline configuration and no failures. The second case included a stuck left ruddervator fixed at the trim setting. The third case included an additional latency added in the command path. Flight conditions were similar for each case, and a summary is given in Table 3.

The pilot was instructed to minimize his inputs while the wavetrains were engaged; any additional inputs should be low in amplitude and frequency, and should be applied only to keep the aircraft from nearing test limits. Measurements were passed through a fourth-order Butterworth high-pass digital filter with a 0.01 $\mathrm{Hz}$ cutoff frequency to effectively detrend the data before applying the Fourier transform. A $20 \mathrm{~s}$ sliding window was used for the recursive Fourier transform, as in Eq. (9), during each maneuver.

To check and compare the real-time results, a post-flight analysis using output error (OE) parameter estimation was performed, as described in Refs. [20,26]. Although this analysis used the same flight data as in the real-time analysis, perturbation values were computed by subtracting trim values, and frequencydomain data was obtained using a a high-accuracy Chirp Z-transform. ${ }^{27,12}$ The analysis frequencies for the transform were $0.125 \mathrm{~Hz}$ to $1.6 \mathrm{~Hz}$, taken in $0.0125 \mathrm{~Hz}$ increments, which is finer than what was used in the real-time analysis. Although computed and visible in Fig. 2, frequency response estimates in this section are not shown for the lowest excitation frequency of $0.050 \mathrm{~Hz}$. The OE results are well approximated in this bandwidth using only the short period approximation, which is of primary interest for these fault scenarios. The Gauss-Newton optimizer in SIDPAC was used to determine the dimensional stability and control derivatives in Eq. (3), with initial values obtained from an equation-error analysis. Parameter estimates and standard errors using output error are given in Table 4 for each maneuver. 
The angle of attack value used in the real-time analysis was the average of the measurements from the left and right vanes. From comparing these time histories, it appeared that the left vane had appreciable shaft friction, which then manifested in the $\alpha / \delta_{\text {lon }}$ frequency response estimates as additional phase lag. For the post-flight OE analysis, an additional parameter to the LOES model, $\tau_{\alpha}$, was added to account for this delay. Alternatives such as time-shifting the data, using only the right-wing measurement, or discarding the angle of attack measurement were considered but not pursued in order to use the same measurements in both analyses.

\section{IV.A. Baseline Configuration}

The first case included the wavetrain excitation during straight and level trimmed flight. Output measurements are shown in Fig. 4. Responses were good for linear modeling, having signal-to-noise ratios above 15 and small perturbations about the trim condition: approximately 2 deg angle of attack, $5 \mathrm{deg} / \mathrm{s} \mathrm{pitch}$ rate, and $0.2 \mathrm{~g}$ units of vertical acceleration. Airspeed varied between $89 \mathrm{ft} / \mathrm{s}$ and $112 \mathrm{ft} / \mathrm{s}$, and ramifications of this are discussed in the next section. Added inputs from the pilot were small in amplitude, comprising less than $1 \%$ of the available travel, and were low in frequency.

Output error estimates were reasonable and had small uncertainties, under $10 \%$ of the parameter values, which indicated the short period approximation was adequate for this data. Estimates of the neglected $L_{q}$ and $L_{\delta_{l o n}}$ parameters were also reasonable, but were not retained because the minor gains in fit did not justify the additional uncertainty in the remaining parameters. The equivalent time delay estimate is reasonable for computation and communication delays involved with remotely flying this aircraft, and the angle of attack measurement delay is consistent with a post-flight data compatibility analysis.

Frequency response estimates from the post-flight OE analysis and the real-time method, obtained at the end of the maneuver, are shown as a Bode plot in Fig. 5. Note again that for a more direct comparison between the real-time and post-flight analyses, the frequency response estimates at $0.050 \mathrm{~Hz}$ are not shown. Some differences between the two results can be seen, particularly in the Bode magnitude, but in general the two methods show good agreement. A phase margin is observed within the frequencies analyzed for the $a_{z} / \delta_{l o n}$ frequency response. Because of this margin, the $a_{z} / \delta_{l o n}$ transfer function is of particular interest and will be examined in the cases with simulated faults.

Time histories of the real-time frequency response estimates are shown in Fig. 6. A copy of Fig. 5 is overlaid on this plot at the maneuver terminus. Estimates were not started until an integer multiple of the corresponding half period was reached, resulting in an initial staggering of the estimates in time. Frequency responses were also updated at these multiples, resembling a zero-order-hold type process. Estimates exhibited large variations at the start of the maneuver, as there was little information content and transient responses in the data. As more data was collected and transient response measurements left the sliding window, responses quickly converged.

\section{IV.B. Ruddervator Fault}

The second maneuver began as the first, but a fault was engaged at $9.36 \mathrm{~s}$ which fixed the left ruddervator to its trim state at the beginning of the maneuver. Output measurements are shown in Fig. 7. Responses were similar to those in Fig. 4 until the fault, and then different afterwards. One difference was due to the changed trim condition. The stuck position of the left ruddervator created more negative lift on the tail and resulted in approximately a 2 deg increase in the angle of attack and a $6 \mathrm{ft} / \mathrm{s}$ decrease in the mean airspeed. Because ruddervators contain both longitudinal and directional commands, the failure of one surface creates a cross-axis coupling in the responses. This was seen in the additional frequency content of the off-axis responses at the pedal input frequencies, and a gain increase for the cross-axis frequency responses (not shown).

Figure $8(\mathrm{a})$ shows the $a_{z} / \delta_{\text {lon }}$ frequency response estimates. The $\mathrm{OE}$ analysis was applied separately to pre-fault and post-fault data. The estimated Bode phase remained approximately the same between the two segments, whereas the Bode gain dropped about $6 \mathrm{~dB}$. This observation is explained by the halving of $M_{\delta_{\text {lon }}}$ due to the faulted left ruddervator, which is statistically consistent with the parameter estimates in Table 4. The increased uncertainties after the fault is a result of unmodeled dynamics present in the data, due to longitudinal responses created by directional inputs from the cross-axis coupling. Despite the model structure error incurred from only using the short period approximation, expected results were still obtained, albeit with higher uncertainty. The real-time estimates, obtained at the time the fault was engaged, match 


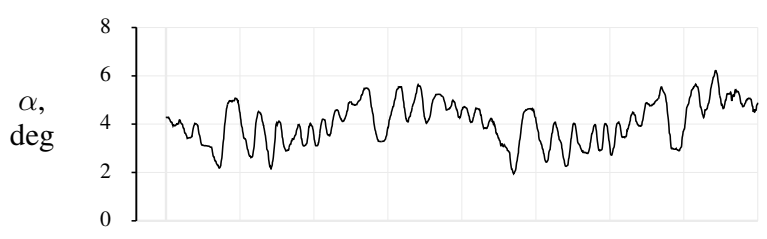
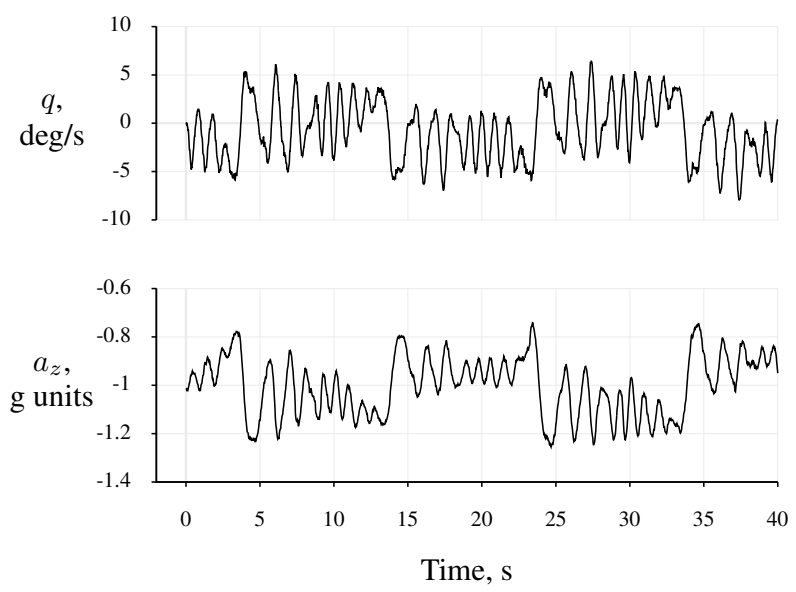

Figure 4. Output measurements, Maneuver 1

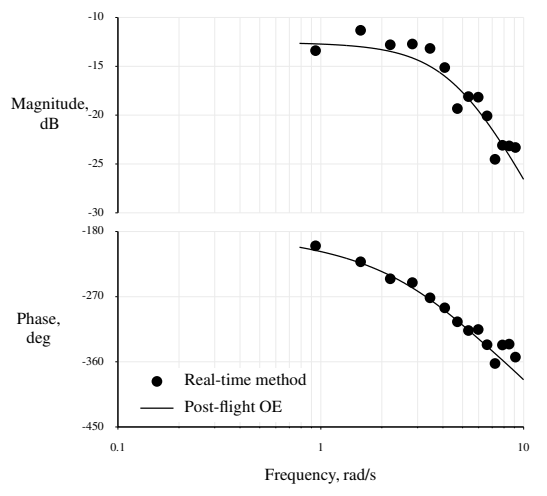

(a) $\alpha / \delta_{l o n}$

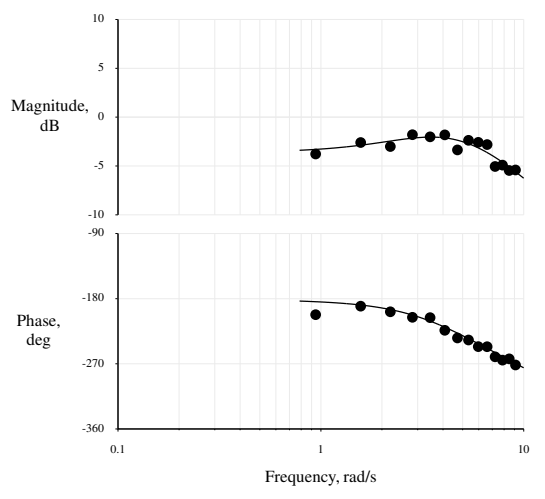

(b) $q / \delta_{\text {lon }}$

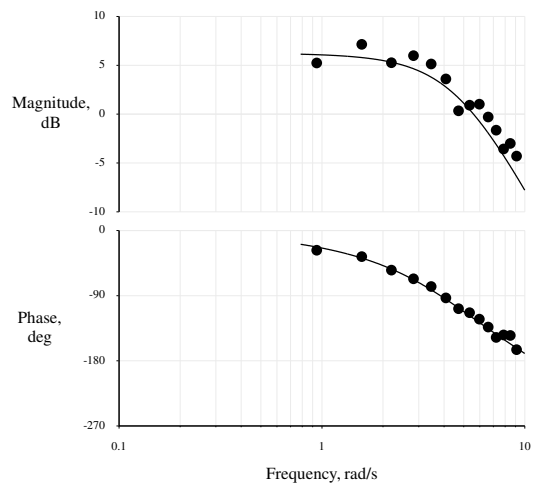

(c) $a_{z} / \delta_{l o n}$

Figure 5. Bode plot results at $t=40 \mathrm{~s}$, Maneuver 1

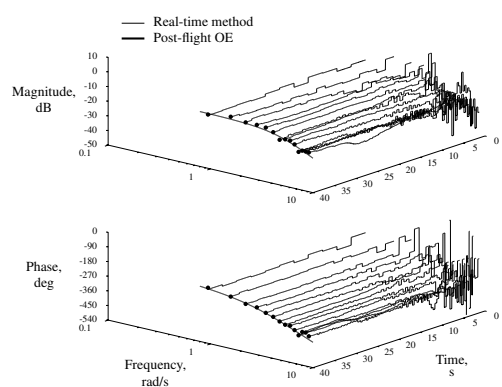

(a) $\alpha / \delta_{l o n}$
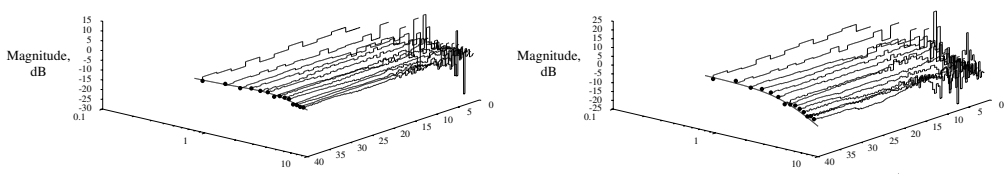

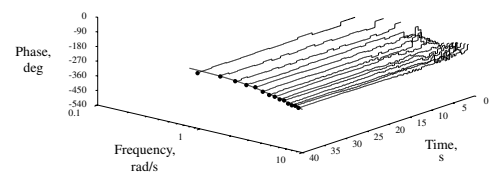

(b) $q / \delta_{\text {lon }}$

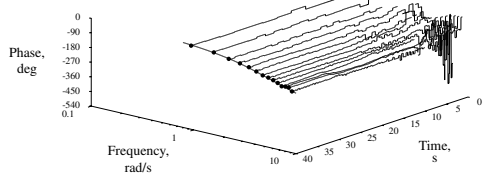

(c) $a_{z} / \delta_{l o n}$

Figure 6. Bode plot history, Maneuver 1 
the OE results well. A noticeable drop in the gain is observed at the end of the end of the maneuver, whereas the phase remained approximately the same.

The effects of the fault on the real-time results are perhaps easier to track using the temporal evolution of a scalar quantity such as the phase margin, shown for this transfer function in Fig. 8(b). Before the fault, the frequency response estimate was settling on a phase margin estimate of $50 \mathrm{deg}$ at $6.4 \mathrm{rad} / \mathrm{s}$, similar to the $\mathrm{OE}$ results and those from the first maneuver. After the fault, the real-time estimates of the crossover frequency decrease and settle around $2.5 \mathrm{rad} / \mathrm{s}$ at $26 \mathrm{~s}$, with a phase margin of about $125 \mathrm{deg}$. The OE estimates follow the same trend, but may be too low in cutoff frequency and too high in phase margin because they extrapolate to lower frequencies where the phugoid mode resides. Also as shown in Table 4, there is more uncertainty in the stability margins due to higher error bounds for the estimated stability and contorl derivatives. However, the correct trend is observed in that a decrease in gain shifts the crossover frequency lower, resulting in a larger phase margin.
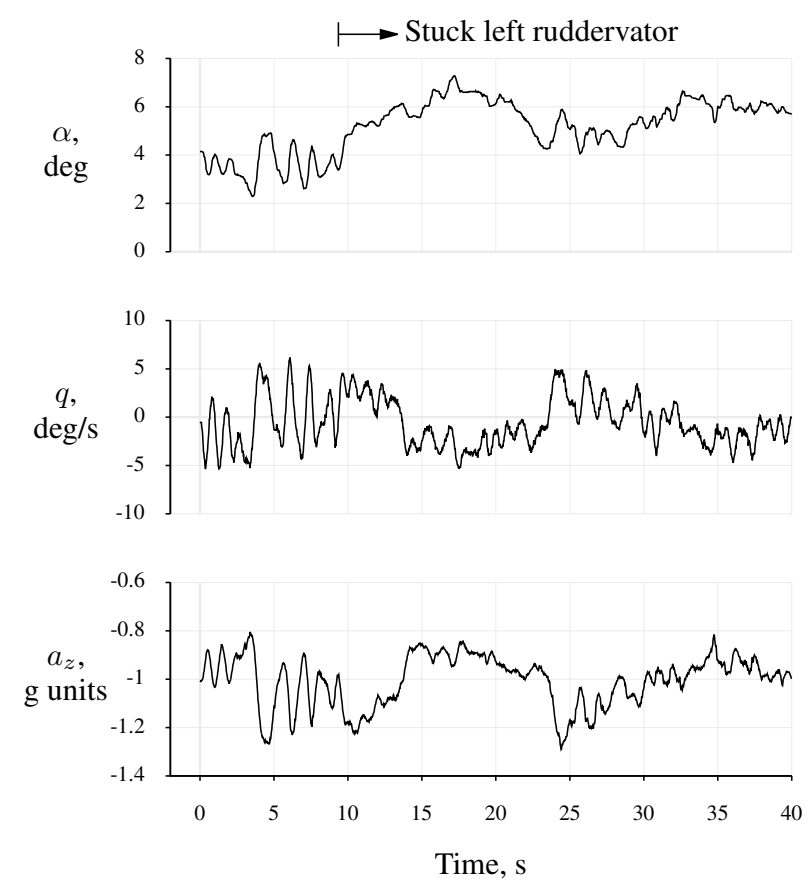

Figure 7. Output measurements, Maneuver 2, with ruddervator fault engaged at $9.36 \mathrm{~s}$

\section{IV.C. Latency Fault}

The third maneuver was similar began as the first, but with a fault engaged at $14.26 \mathrm{~s}$ which added a delay in the command path. This delay began at $0.025 \mathrm{~s}$, and then increased by $0.010 \mathrm{~s}$ every $5 \mathrm{~s}$ until the end of the maneuver, finishing at $0.075 \mathrm{~s}$. Output measurements are shown in Fig. 9, which are only slightly distinguishable from those shown in Fig. 4.

Figure 10(a) shows the Bode plot estimates. There was not enough information content to reliably estimate parameters for each $5 \mathrm{~s}$ post-fault segment, even when including prior information from previous data segments. Therefore the maneuver was analyzed separately as a pre-fault segment and a post-fault segment, as before. Estimation results were reasonable, and a $0.036 \mathrm{~s}$ added delay was observed, which approximately corresponds to the average time delay for the post-fault data. The OE estimates in Fig. 10(a) show approximately the same Bode magnitude, and a small shift in the Bode phase due to the added delay. Uncertainty estimates for the OE estimates given in Table 4 indicate accurate estimates of the model terms. Similar results are observed in the real-time estimates.

Time histories of phase margin estimates are shown in Fig. 10(b). The OE models result in crossover frequencies of about $5.6 \mathrm{rad} / \mathrm{s}$, and phase margins of $58 \mathrm{deg}$ before the fault and $45 \mathrm{deg}$ after the fault. The real-time results converged on $6.3 \mathrm{rad} / \mathrm{s}$ at $10 \mathrm{~s}$, and then do not vary much until the end of the maneuver, except for a few transients when the fault is engaged. The phase margin also converged after $10 \mathrm{~s}$ at 47 deg, and then decreased to 26 deg by the end of the maneuver. Each result follows the expected result that 


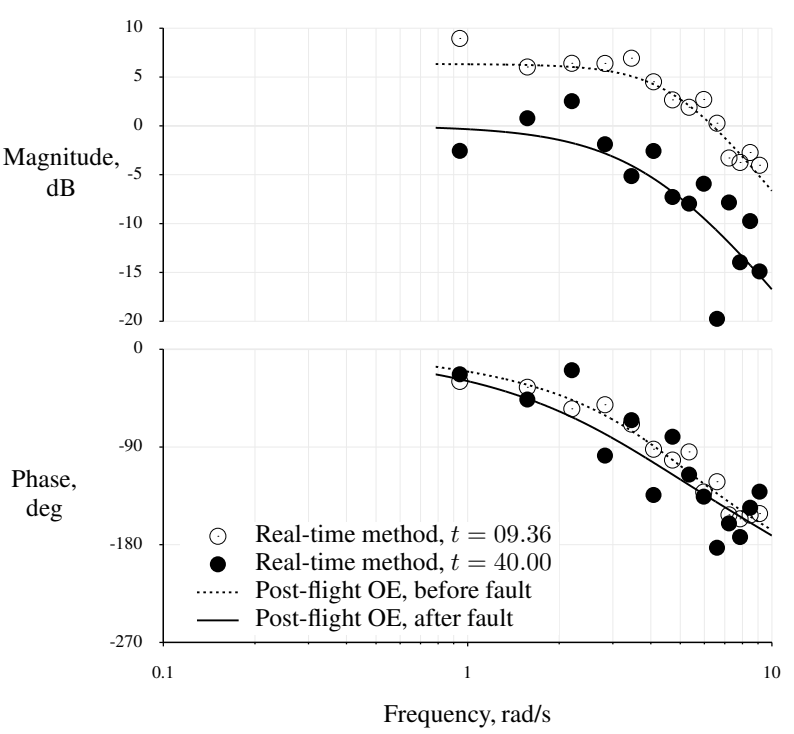

(a) Bode plots

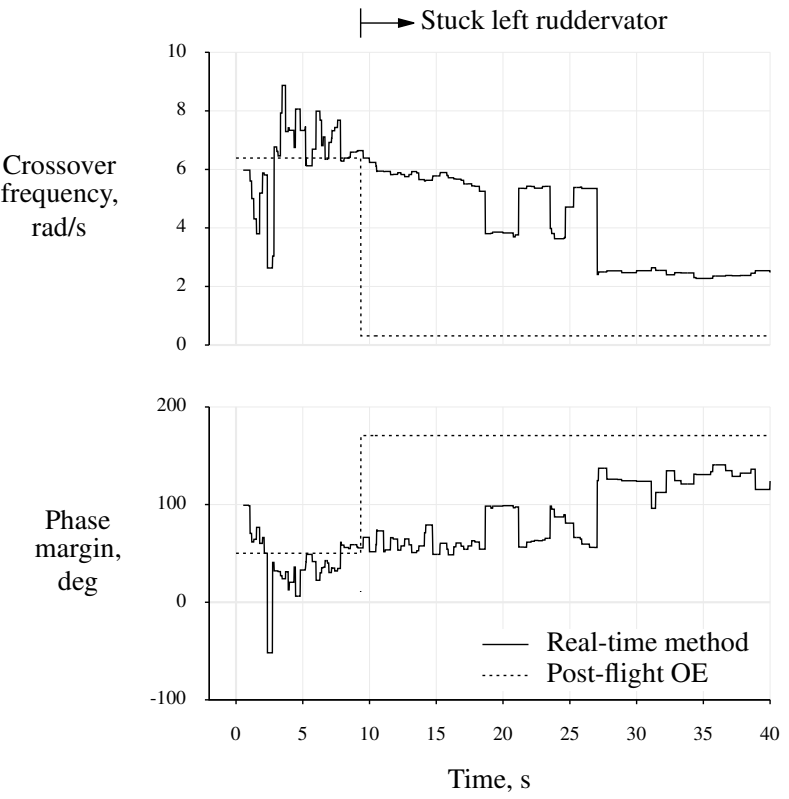

(b) phase margin history

Figure 8. Results for $a_{z} / \delta_{l o n}$, Maneuver 2, with ruddervator fault engaged at $9.36 \mathrm{~s}$

an increase in delay creates faster roll-off in the Bode phase, thereby reducing the phase margin for a given crossover frequency.

\section{Discussion of Results}

This section elaborates on a few observations seen in the results. The first is that the real-time estimates seem to exhibit some undesirable errors when compared to the OE estimates. This is despite good perturbation signals, signal-to-noise ratios, low turbulence levels, and good theoretical convergence properties that were demonstrated previously using flight test data. ${ }^{11}$ These errors were found to be due to the relatively large variation in airspeed and dynamic pressure that resulted from exciting the phugoid mode. Specifically, airspeed changes about $12 \%$ and dynamic pressure changed about $24 \%$ during the maneuvers. These changes violate the constant conditions inherently assumed in frequency response modeling. For reference, it is recommended that no more than $10 \%$ variation in dynamic pressure can be tolerated when applying output error with time-domain data for linear time-invariant models. ${ }^{28}$ Dimensional stability and control derivatives include dynamic pressure effects, so that models using these parameters are sensitive to variations in dynamic pressure. Other variations such as mass distribution and engine speed were under $0.05 \%$ and $5 \%$, respectively, which were negligible.

To investigate the extent of the effect of changing airspeed and dynamic pressure on the real-time estimation results, a separate analysis of Maneuver 1 was conducted. A state-space model of the short-period approximation, similar to Eq. (2), was used to estimate the nondimensional stability and control derivatives using an equation error analysis in the frequency domain. Values from the second column in Table 4 were used for the time delays. Bode plots for the $a_{z} / \delta_{l o n}$ transfer function were then computed for the trim condition of Maneuver 1, but also for its maximum and minimum dynamic pressures. These plots are shown in Fig. 11. The nominal transfer function is in good agreement with that shown in Fig. 5. A 2 dB variation, corresponding to a roughly $25 \%$ change in magnitude of the $a_{z}$ response, is seen in the Bode gain due to the changes in airspeed and dynamic pressure. This is a significant variation, and can explain the scatter seen in the real-time estimates, as compared with theory, simulation data, and previous results. The Bode phase shows little variation, which is also consistent with the results presented. A slightly larger variation is expected in Maneuver 2 due to larger variations in airspeed and dynamic pressure caused by the ruddervator fault. A fourth maneuver (not shown), similar to the first maneuver but with $30 \%$ smaller input amplitudes, exhibited only $5 \%$ variation in airspeed and $11 \%$ in dynamic pressure, and resulted in frequency responses 

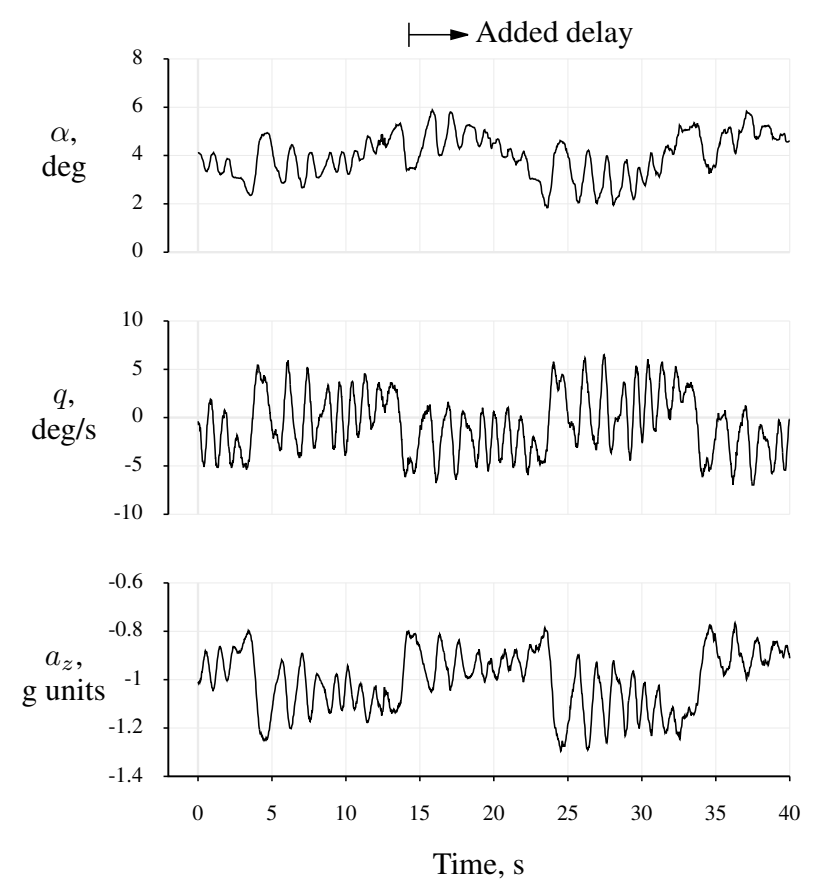

Figure 9. Output measurements, Maneuver 3, with latency fault engaged at $14.26 \mathrm{~s}$

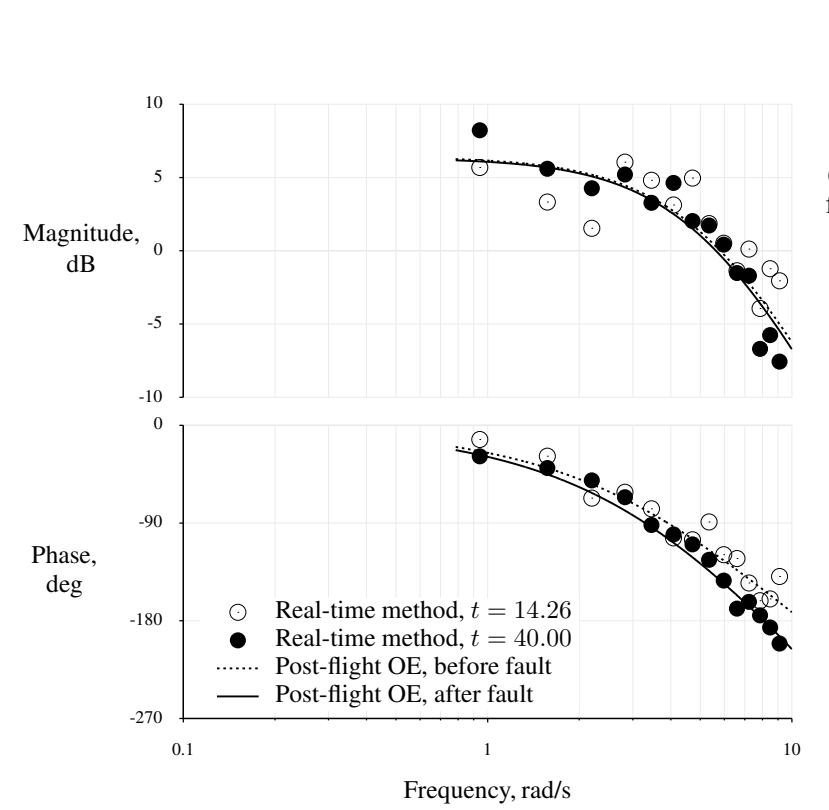

(a) Bode plots

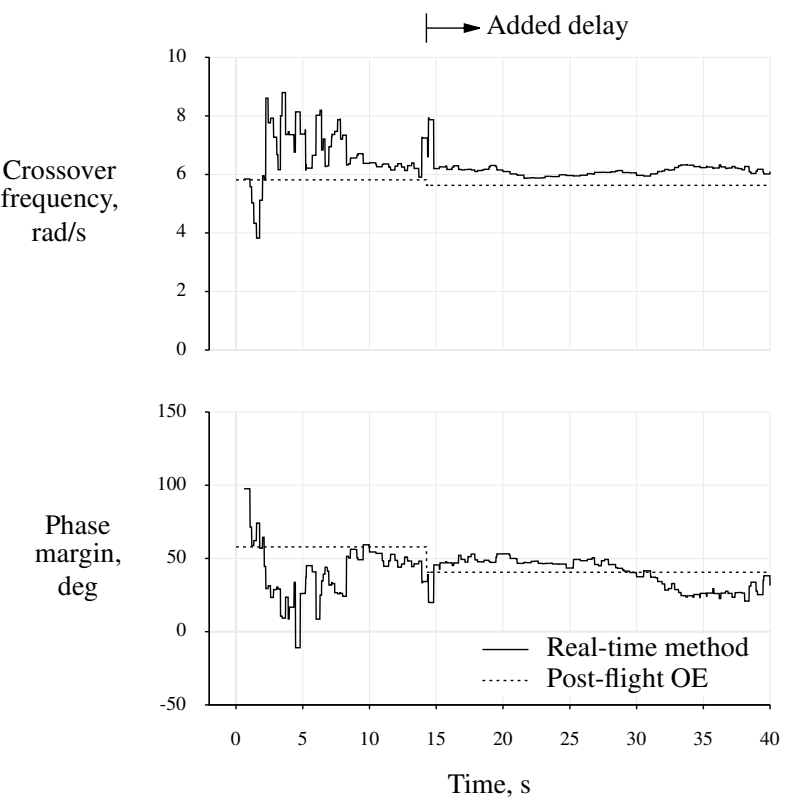

(b) phase margin history

Figure 10. Results for $a_{z} / \delta_{l o n}$, Maneuver 3, with latency fault engaged at $14.26 \mathrm{~s}$ 
more closely aligned with the OE estimates, which also had smaller uncertainty levels on the estimated parameters. A redesign of the multisine input, having knowledge about the phugoid mode, would either attenuate or discard the lowest frequencies.

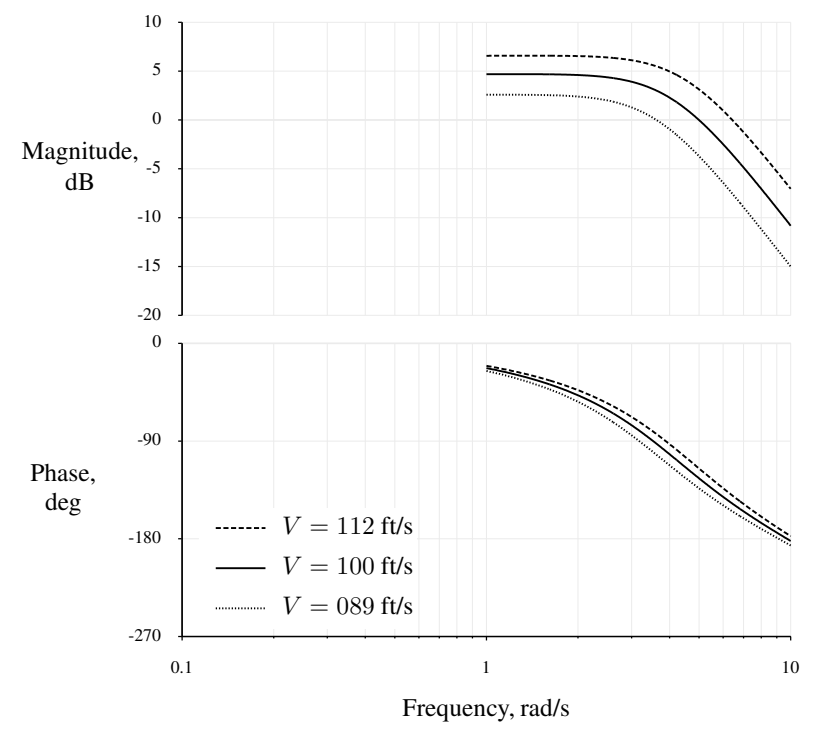

Figure 11. Variation in $a_{z} / \delta_{l o n}$ due to dynamic pressure, Maneuver 1

Another source of error in the real-time estimates stems from nonlinear elements in the system, which add harmonics that distort the frequency response. One such element was the the command shaping filter just downstream of the pilot inputs. Figure 12 shows a frequency response estimate of the filter, using the measured inputs for Maneuver 1 and the outputs of the filter, which were computed using the known filter equations. Analytical linearization of the filter equations using the root mean square value of the inputs as the perturbation size results in an expected $20 \mathrm{~dB}$ gain, which is confirmed by Fig. 12 . Frequencydependent distortions to this result are seen in both the Bode gain and phase plots. These distortions are then propagated downstream, and affect the resulting stick-to-response frequency response estimates. Using inputs downstream of the command shaper resulted in slightly tighter frequency response estimates and were considered, but were not pursued because they altered the equivalent time delay estimates.

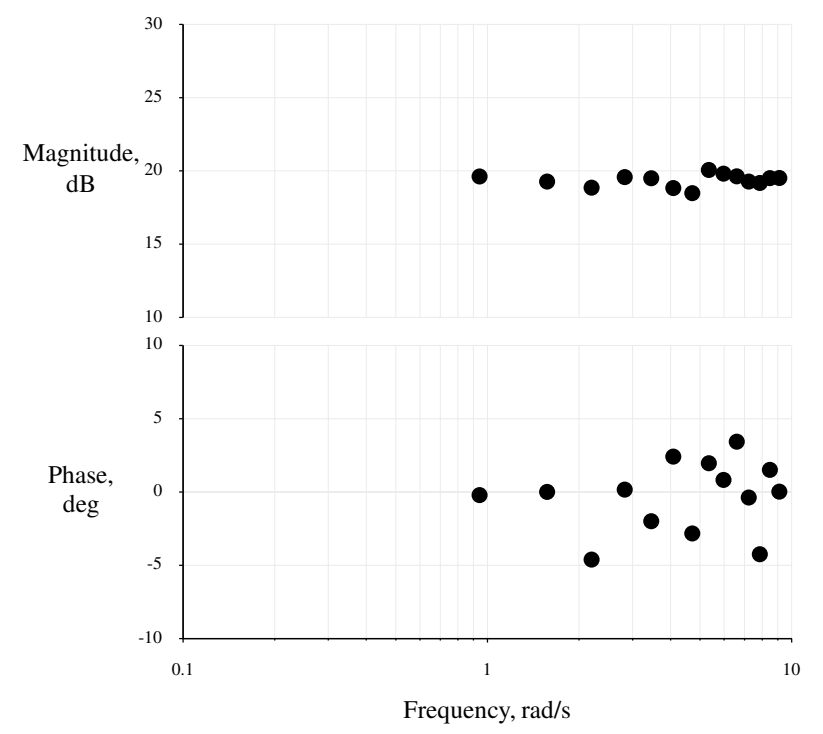

Figure 12. Frequency response estimates of longitudinal stick shaper, Maneuver 1

There is perhaps a tendency when examining the Bode plots in the previous section to trust the OE results more than the real-time estimates, due to the smooth frequency responses shown. This may be misleading 
because the $\mathrm{OE}$ method is estimating parameters in a specified dynamic model. Uncertainties in the data and model structure manifest as degraded fits to data, biased parameters, and increased uncertainty levels, as in Maneuver 2. As elaborated in Ref. [20], parameter estimates in Eq. (3) are correlated and therefore more difficult to discern. In this light, the real-time method can potentially provide a simpler and more accurate measure of the frequency response. No model structure needs to be selected in this type of analysis. If the experiment abides by the proper assumptions, the real-time estimates have excellent theoretical properties. Estimates at each frequency depend only on the data at that frequency, whereas the model parameters estimated by the OE method have wide-band sensitivities.

A $20 \mathrm{~s}$ sliding window of data was used for the real-time estimates. This type and length was selected to demonstrate an adequate trade-off between accuracy and adaptation within the span on one $40 \mathrm{~s}$ maneuver. Shorter window lengths and faster adaptation rates are possible at the expense of less data information content. ${ }^{11}$ Frequency-dependent windows or other windowing functions may be beneficial, at the cost of added complexity. An exponential forgetting method ${ }^{29}$ has been attempted in the past, ${ }^{11}$ but has displayed poorer performance than a sliding window for frequency response estimation. Selection of the best dataforgetting method for a particular situation is an open problem.

This study showed that a real-time modeling method could be used to identify faults in straight and level flight. Aircraft in serious loss-of-control situations can experience nonlinear motions with rapidly-varying flight conditions. In these cases it may not be possible to excite small perturbation maneuvers over $10 \mathrm{~s}$ or $20 \mathrm{~s}$ and achieve a reasonable frequency response estimate. It would be recommended then to only attempt to excite short period, dutch roll, and roll modes to get a rough idea of time delays and if control surfaces were stuck or damaged. Another possibility is that there could be enough excitation in the aircraft already to estimate reasonable frequency responses, but this case is perhaps better analyzed using techniques that employ averaging.

However, there are cases when this method is very applicable. One case is for aircraft that develop icing, which occurs slowly over time and in steady conditions. A short multisine input, eliciting responses similar to moderate turbulence, could identify faults with only minor irritation to occupants. Detection of changing dynamics can be accomplished through monitoring stability margins and crossover frequencies. This type of stability margin monitoring could have been useful for the study described in Ref. [4], where pilot-induced oscillations were encountered as a result of the nonlinear adaptive controller.

\section{Concluding Remarks}

A method for real-time frequency response estimation from measured time histories of input and output data was demonstrated. Orthogonal phase-optimized multisine inputs were simultaneously applied to the pilot longitudinal stick, lateral stick, and pedal inputs. A recursive Fourier transform updated frequency domain data, and a simple method was applied for computing frequency response functions and stability margins. A $20 \mathrm{~s}$ sliding window of data was used to facilitate identification of time-varying dynamics. No model structure was required, and the only assumption involved was that the dynamics could be usefully approximated as linear.

Three cases were investigated: straight and level flight, a stuck left ruddervator, and an added command path latency. In each case, frequency response estimates adapted to the faults in the expected manners. Results were in good agreement with those determined using a post-flight output-error analysis with a loworder equivalent system model. The main differences were found to be due to a relatively large oscillation in dynamic pressure, caused by excitation of the phugoid mode. To a lesser degree, differences are also due to the nonlinear command shaper downstream of the pilot inputs.

This method is well suited to real-time modeling situations where a model structure may not be known prior to flight testing. Some examples include flying new aircraft, such as in this study, or finding loworder models of higher-order closed-loop aircraft. Another example is tracking stability margins for adaptive control laws or time-varying pilot models. If multisine inputs could be applied, these techniques could also be leveraged for detecting faults or changing dynamics, and thereby avoiding the onset of loss-of-control situations. One advantage of this approach is that it relies only on measured data, and is applied in a straightforward manner without introducing engineering judgment into the analysis. 


\section{Acknowledgments}

The efforts of the AirSTAR flight test team at NASA Langley Research Center in modifying and testing the Bat-4 aircraft and associated systems, calibrating the instrumentation, and carrying out the flight operations are gratefully acknowledged. Mr. Daniel Murri of NASA Langley Research Center and NASA Engineering and Safety Center served as the research pilot. Flight tests were conducted at NASA Wallops Flight Facility. The system identification research was supported by the NASA Aeronautics Research Mission Directorate (ARMD) Learn-To-Fly project. 


\section{References}

${ }^{1}$ Belcastro, C. and Foster, J., "Aircraft Loss-of-Control Accident Analysis," No. 2010-8004, AIAA, Toronto, Ontario Canada, August 2010.

${ }^{2}$ Menke, T. and Maybeck, P., "Sensor/Actuator Failure Detection in the Vista F-16 by Multiple Model Adaptive Estimation," IEEE Transactions on Aerospace and Electronic Systems, Vol. 31, No. 4, October 1995, pp. 1218-1229.

${ }^{3}$ Kobayashi, T. and Simon, D., "Application of a Bank of Kalman Filters for Aircraft Engine Fault Diagnostics," Tech. Rep. TM-2003-212526, NASA, Cleveland, OH, August 2003.

${ }^{4}$ Bosworth, J. and Williams-Hayes, P., "Flight Test Results from the NF-15B Intelligent Flight Control System (IFCS) Project with Adaptation to a Simulated Stabilator Failure," Tech. Rep. TM-2007-214629, NASA, Edwards, CA, December 2007.

${ }^{5}$ Balough, D., "Determination of X-36 Stability Margins Using Real-Time Frequency Response Techniques," No. 1998-4154 in Atmospheric Flight Mechanics Conference, AIAA, Boston, MA, 1998.

${ }^{6}$ Sahai, R., Tischler, M., Blanken, C., Sullivan, C., , Wei, M., Ng, Y.-S., and Pierce, L., "Flight-Time Identification of Helicopter-Slug Load Frequency Response Characteristics Using CIFER ${ }^{\circledR}$," No. 1999-4246 in Atmospheric Flight Mechanics Conference, AIAA, Portland, OR, 1999.

${ }^{7}$ Zaal, P. and Sweet, B., "Estimation of Time-Varying Pilot Model Parameters," No. 2011-6474 in Modeling and Simulation Technologies Conference, AIAA, Portland, OR, August 2011.

${ }^{8}$ Thompson, P., Klyde, D., Bachelder, E., Rosenthal, T., and Brenner, M., "Development of Wavelet-Based Techniques for Detecting Loss of Control," No. 2004-5064 in Atmospheric Flight Mechanics Conference, AIAA, Providence, RI, August 2004.

${ }^{9}$ Klyde, D., Thompson, P., Bachelder, E., and Rosenthal, T., "Evaluation of Wavelet-Based Techniques for Detecting Loss of Control," No. 2004-4702 in Atmospheric Flight Mechanics Conference, AIAA, Providence, RI, August 2004.

${ }^{10}$ Lichter, M., Bateman, A., and Balas, G., "Flight Test Evaluation of a Run-Time Stability Margin Estimation Tool," No. 2009-6257 in Guidance, Navigation, and Control Conference, AIAA, Chicago, IL, August 2009.

${ }^{11}$ Grauer, J. and Morelli, E., "Method for Real-Time Frequnecy Response and Uncertainty Estimation," Journal of Guidance, Control, and Dynamics, Vol. 37, No. 1, January-February 2014, pp. 336-343.

${ }^{12}$ Klein, V. and Morelli, E., Aircraft System Identification: Theory and Practice, AIAA Education Series, AIAA, Reston, VA, 2006.

${ }^{13}$ Cunningham, K., Cox, D., Riddick, S., and Laughter, S., "AirSTAR Beyond Visual Range System Description and Preliminary Test Results," No. 2016-???? in Guidance, Navigation, and Control Conference, AIAA, San Diego, CA, January 2016.

${ }^{14}$ Cunningham, K., Foster, J., Morelli, E., and Murch, A., "Practical Application of a Subscale Transport Aircraft for Flight Research in Control Upset and Failure Conditions," No. 2008-6200 in Atmospheric Flight Mechanics Conference, AIAA, Honolulu, HI, August 2008.

${ }^{15}$ Morelli, E., "Real-Time Global Nonlinear Aerodynamic Modeling for Learn-To-Fly," No. 2016-???? in Atmospheric Flight Mechanics Conference, AIAA, San Diego, CA, January 2016.

${ }^{16}$ Morelli, E., "Multiple Input Design for Real-Time Parameter Estimation in the Frequency Domain," No. REG-360 in $13^{\text {th }}$ IFAC Symposium on System Identification, IFAC, Rotterdam, The Netherlands, 2003.

${ }^{17}$ Morelli, E., "Flight Test Experiment Design for Characterizing Stability and Control of Hypersonic Vehicles," Journal of Guidance, Control, and Dynamics, Vol. 32, No. 3, May-June 2009, pp. 949-959.

${ }^{18}$ McRuer, D., Ashkenas, I., and Graham, D., Aircraft Dynamics and Automatic Control, Pinceton University Press, Princeton, NJ, 1973.

${ }^{19}$ Hodgkinson, J., Aircraft Handling Qualities, AIAA Education Series, AIAA, Reston, VA, 1990.

${ }^{20}$ Morelli, E., "Identification of Low Order Equivalent Systems from Flight Test Data," Tech. Rep. TM-2000-210117, NASA, Hampton, VA, August 20002000.

${ }^{21}$ Norton, J., An Introduction to System Identification, Academic Press, London, England, 1986.

${ }^{22}$ Ljung, L., System Identification: Theory for the User, Information and System Sciences Series, Prentice Hall, Upper Saddle River, NJ, 2nd ed., 1990.

${ }^{23}$ Bendat, J. and Piersol, A., Random Data: Analysis and Measurement Procedures, Wiley, Hoboken, NJ, 4th ed., 2010.

${ }^{24}$ Rake, H., "Step Response and Frequency Response Methods," Automatica, Vol. 16, No. 5, September 1980, pp. 519-526.

${ }^{25}$ Jenkins, G. and Watts, D., Spectral Analysis and its Applications, Series in Time Series Analysis, Holden-Day, San Francisco, CA, 1968.

${ }^{26}$ Morelli, E., "Low-Order Equivalent System Identification for the Tu-144LL Supersonic Transport Aircraft," Journal of Guidance, Control, and Dynamics, Vol. 26, No. 2, March-April 2003, pp. 354-362.

${ }^{27}$ Morelli, E., "High Accuracy Evaluation of the Finite Fourier Transform Using Sampled Data," Tech. Rep. TM-110340, NASA, Hampton, VA, June 1997.

${ }^{28}$ Maine, R. and Iliff, K., "Application of Parameter Estimation to Aircraft Stability and Control: The Output-Error Approach," Tech. Rep. RP-1168, NASA, Edwards, CA, June 1986.

${ }^{29}$ Morelli, E. and Smith, M., "Real-Time Dynamic Modeling: Data Information Requirements and Flight-Test Results," Journal of Aircraft, Vol. 46, No. 6, November-December 2009, pp. 1894-1905. 


\section{Tables}

Table 1. Bat-4 geometry and nominal mass properties

\begin{tabular}{ccc}
\hline \hline Parameter & Value & Unit \\
\hline$b$ & 12.68 & $\mathrm{ft}$ \\
$\bar{c}$ & 1.500 & $\mathrm{ft}$ \\
$S$ & 19.02 & $\mathrm{ft}^{2}$ \\
$x_{c m}$ & 48.98 & in \\
$y_{c m}$ & 0.036 & in \\
$z_{c m}$ & 24.01 & in \\
$m$ & 3.593 & slug \\
$I_{x x}$ & 9.841 & slug $\cdot \mathrm{ft}^{2}$ \\
$I_{y y}$ & 8.861 & slug $\cdot \mathrm{ft}^{2}$ \\
$I_{z z}$ & 16.17 & slug. $\mathrm{ft}^{2}$ \\
$I_{x z}$ & -1.015 & slug. $\cdot \mathrm{ft}^{2}$ \\
\hline \hline
\end{tabular}

17 of 19 
Table 2. Multisine inputs, Wavetrain $21(T=40 \mathrm{~s})$

\begin{tabular}{|c|c|c|c|c|}
\hline Input & $k$ & $f_{k}, \mathrm{~Hz}$ & $a_{k}, \operatorname{deg}$ & $\phi_{k}, \mathrm{rad}$ \\
\hline & 2 & 0.050 & 0.026 & +0.391 \\
\hline & 6 & 0.150 & 0.026 & +0.130 \\
\hline & 10 & 0.250 & 0.026 & -2.111 \\
\hline & 14 & 0.350 & 0.026 & +2.126 \\
\hline & 18 & 0.450 & 0.026 & -0.422 \\
\hline & 22 & 0.550 & 0.026 & -2.764 \\
\hline$\delta_{l o n}$ & 26 & 0.650 & 0.026 & +0.224 \\
\hline$a=0.1$ & 30 & 0.750 & 0.026 & +2.244 \\
\hline \multirow[t]{13}{*}{$\mathrm{RPF}=1.044$} & 34 & 0.850 & 0.026 & -2.414 \\
\hline & 38 & 0.950 & 0.026 & +0.319 \\
\hline & 42 & 1.050 & 0.026 & +0.023 \\
\hline & 46 & 1.150 & 0.026 & -0.478 \\
\hline & 50 & 1.250 & 0.026 & -0.473 \\
\hline & 54 & 1.350 & 0.026 & -0.472 \\
\hline & 58 & 1.450 & 0.026 & +1.335 \\
\hline & 3 & 0.075 & 0.026 & -0.702 \\
\hline & 7 & 0.175 & 0.026 & +0.009 \\
\hline & 11 & 0.275 & 0.026 & +0.438 \\
\hline & 15 & 0.375 & 0.026 & -1.935 \\
\hline & 19 & 0.475 & 0.026 & -1.766 \\
\hline & 23 & 0.575 & 0.026 & -0.390 \\
\hline$\delta_{l a t}$ & 27 & 0.675 & 0.026 & +1.456 \\
\hline$a=0.1$ & 31 & 0.775 & 0.026 & +1.608 \\
\hline \multirow[t]{13}{*}{$\mathrm{RPF}=1.185$} & 35 & 0.875 & 0.026 & +0.272 \\
\hline & 39 & 0.975 & 0.026 & +1.864 \\
\hline & 43 & 1.075 & 0.026 & -2.277 \\
\hline & 47 & 1.175 & 0.026 & +1.018 \\
\hline & 51 & 1.275 & 0.026 & -2.391 \\
\hline & 55 & 1.375 & 0.026 & +0.607 \\
\hline & 59 & 1.475 & 0.026 & -0.758 \\
\hline & 5 & 0.125 & 0.026 & -2.690 \\
\hline & 9 & 0.225 & 0.026 & +0.7155 \\
\hline & 13 & 0.325 & 0.026 & +1.690 \\
\hline & 17 & 0.425 & 0.026 & +3.012 \\
\hline & 21 & 0.525 & 0.026 & +1.369 \\
\hline & 25 & 0.625 & 0.026 & +3.134 \\
\hline$\delta_{p e d}$ & 29 & 0.725 & 0.026 & -2.415 \\
\hline$a=0.1$ & 33 & 0.825 & 0.026 & -0.609 \\
\hline \multirow[t]{7}{*}{$\mathrm{RPF}=1.186$} & 37 & 0.925 & 0.026 & -2.585 \\
\hline & 41 & 1.025 & 0.026 & +1.257 \\
\hline & 45 & 1.125 & 0.026 & -1.770 \\
\hline & 49 & 1.225 & 0.026 & -0.836 \\
\hline & 53 & 1.325 & 0.026 & -2.329 \\
\hline & 57 & 1.425 & 0.026 & +2.359 \\
\hline & 61 & 1.525 & 0.026 & +2.913 \\
\hline
\end{tabular}

18 of 19 
Table 3. Maneuver and flight condition summary

\begin{tabular}{cccccccc}
\hline \hline No. & Description & $V, \mathrm{ft} / \mathrm{s}$ & $\alpha, \mathrm{deg}$ & $h, \mathrm{ft}$ & $m$, slug & $x_{c m}$, in & $I_{y y}, \mathrm{slug} \cdot \mathrm{ft}^{2}$ \\
\hline 1 & straight and level flight & 100.1 & 4.301 & 4001 & 3.525 & 49.03 & 8.857 \\
2 & stuck left ruddervator & 100.2 & 4.141 & 3970 & 3.502 & 49.04 & 8.852 \\
3 & added command latency & 99.97 & 4.091 & 4010 & 3.499 & 49.04 & 8.852 \\
\hline \hline
\end{tabular}

Table 4. Output error arameter estimates and standard errors

\begin{tabular}{cccccc}
\hline \hline Parameter & \multirow{2}{*}{ Maneuver 1 } & \multicolumn{2}{c}{ Maneuver 2 } & \multicolumn{2}{c}{ Maneuver 3 } \\
& & Before fault & After fault & Before fault & After fault \\
\hline$L_{\alpha}$ & $+2.797 \pm 0.056$ & $+3.298 \pm 0.071$ & $+2.801 \pm 0.300$ & $+2.999 \pm 0.061$ & $+2.786 \pm 0.089$ \\
$M_{\alpha}$ & $-7.669 \pm 0.474$ & $-10.86 \pm 0.421$ & $-3.281 \pm 1.886$ & $-6.647 \pm 0.686$ & $-6.612 \pm 0.852$ \\
$M_{q}$ & $-5.170 \pm 0.357$ & $-3.785 \pm 0.252$ & $-4.620 \pm 1.245$ & $-7.738 \pm 0.627$ & $-5.559 \pm 0.620$ \\
$M_{\delta_{l o n}}$ & $-5.220 \pm 0.216$ & $-4.720 \pm 0.160$ & $-1.867 \pm 0.279$ & $-6.711 \pm 0.378$ & $-5.173 \pm 0.356$ \\
$\tau_{l o n}$ & $+0.062 \pm 0.004$ & $+0.051 \pm 0.004$ & $+0.058 \pm 0.017$ & $+0.086 \pm 0.005$ & $+0.122 \pm 0.007$ \\
$\tau_{\alpha}$ & $+0.061 \pm 0.005$ & $+0.054 \pm 0.007$ & $+0.065 \pm 0.027$ & $+0.065 \pm 0.005$ & $+0.053 \pm 0.008$ \\
\hline$\omega_{s p}$ & 4.704 & 4.832 & 4.028 & 5.464 & 5.275 \\
$\zeta_{s p}$ & 0.847 & 0.733 & 0.921 & 0.983 & 0.971 \\
$T_{\theta_{2}}$ & 2.979 & 3.298 & 2.801 & 2.999 & 2.857 \\
\hline \hline
\end{tabular}

